\title{
EDUCACIÓN: LOS DESAFÍOS DEL APRENDIZAJE EN LÍNEA Y LAS DESIGUALDADES EDUCATIVAS
}

\section{EDUCATION: THE CHALLENGES OF ONLINE LEARNING AND EDUCATIONAL INEQUALITIES}

\author{
Griselda Fabiana Medina $^{1}$ - Clotilde Sogari Noemí ${ }^{2}$
}

Fecha de recepción: 06-06-2020

Fecha de aceptación y versión final: 18-06-2021

\section{Resumen}

La siguiente publicación analiza los conocimientos básicos que presentan los alumnos de los cursos 4to (A y B) y 3ro (A y B) de la Escuela Regional de Agricultura, Ganadería e Industrias afines (ERAGIA) ubicada en la provincia de Corrientes, Argentina. El objetivo es conocer el equipamiento tecnológico del cual disponen los alumnos de acuerdo a sus condiciones socioeconómicas, evaluar los conocimientos que presentan sobre las herramientas básicas de su ordenador, el manejo y uso de las nuevas tecnologías que emergen de una modalidad virtual de enseñanza. La metodología empleada es cuantitativa y exhibe un alcance descriptivo, donde se pretende analizar y recopilar información sobre el uso y manejo que presenta el educando respecto a las nuevas tecnologías, se investiga el equipamiento tecnológico que dispone el grupo clase para crear un nuevo esquema de trabajo a futuro que garantice un sistema de enseñanza virtual de calidad que promueva la construcción de un conocimiento útil, verdadero, significativo y que perdure en el tiempo. Se concluye, que la readaptación y el ajuste de la enseñanza en entornos virtuales requieren de una planificación basada en el desarrollo individual y expansión de habilidades que propicien un escenario optimo en el proceso de enseñanza-aprendizaje. Tanto docentes como alumnos precisan de una alfabetización tecnológica para que puedan acceder y conducirse de manera competente a través de la cultura y tecnología digital.

Palabras clave: aprendizaje- desigualdades educativas- entornos virtuales- enseñanza.

\section{Abstract}

The following publication analyzes the basic knowledge presented by the students of the 4th (A and $B$ ) and 3rd (A and B) courses of the Regional School of Agriculture, Livestock and Industries (ERAGIA) located in the province of Corrientes, Argentina. The objective is to know the technological equipment available to students according to their socioeconomic conditions, evaluate the knowledge they present about the basic tools of their computer, the management and use of new technologies that emerge from a virtual teaching modality. The methodology used is quantitative and exhibits a descriptive scope, where it is intended to analyze and collect

\footnotetext{
${ }^{1}$ Investigadora integrante del grupo de investigación G.I.E.S.M.A de la FaCENA de la Universidad Nacional del Nordeste (UNNE). Profesora en Ciencias Químicas y del Ambiente. Jefe de Trabajo Prácticos en la asignatura Electricidad, Magnetismo, Óptica y Sonido, Facultad de Ciencias Exactas y Naturales y Agrimensura, UNNE. Correo electrónico: gri3783@gmail.com

2 Directora del Grupo de Investigación, Desarrollo y Transferencia Tecnológica de las Energías Renovables y Cuidado del Medio Ambiente. G.I.E.S.M.A de la FaCENA (UNNE). Docente Investigadora Categoría II del Departamento de Física de la Facultad de Ciencias Exactas y Naturales y Agrimensura. Directora del Grupo de Investigación del uso de las Energías Renovables y Cuidado del Medio Ambiente, Co responsable de la coordinación de la Red Iberoamericana de Investigación para la aplicación de las Energías Renovables y Cuidado Ambiental, aprobado por la AUIP en 2017. Correo electrónico: noemisogari@gmail.com
} 
RIIE (2021), Año 12 (15), 44 - 54.

DOI: http://dx.doi.org/10.30972/riie.12155567
Educación: los desafíos del aprendizaje en línea y las desigualdades educativas.

information on the use and management presented by the student regarding new technologies, the technological equipment available to the class group is investigated to create a new work scheme in the future that guarantees a quality virtual teaching system that promotes the construction of useful, true, meaningful knowledge that lasts over time. It is concluded that the readjustment and adjustment of teaching in virtual environments require planning based on individual development and expansion of skills that provide an optimal setting in the teachinglearning process. Both teachers and students need technological literacy so that they can access and conduct themselves competently through culture and digital technology.

Key words: learning- educational inequalities- virtual environments- teaching. 


\section{Introducción}

\section{La Educación y la tecnología como soporte didáctico}

La interrupción de las clases a causa de la pandemia COVID-19, ha forzado el traslado de las clases presenciales a una modalidad virtual de enseñanza mediante el uso de plataformas educativas seleccionadas en función a las condiciones socioeconómicas de los alumnos, que permitan establecer un conjunto de actividades que admita el trabajo individual o grupal, que posibilite la interpretación, la reflexión y la resolución de problemas que promuevan la construcción del conocimiento.

Abreu (2020) expresa que "sorprendentemente esta pandemia ha obligado a todos a experimentar e improvisar con el aprendizaje digital" (p.4). Bajo estas circunstancias el sector educativo se debe reedificar de manera inmediata, garantizando la fiabilidad de la enseñanza en este entorno virtual. Fernández y González (2009) explican que "un entorno virtual de aprendizaje, es un espacio virtual donde se brindan diferentes servicios y herramientas que permiten a los participantes la construcción de conocimiento" (p.3). La adaptación de la enseñanza a este nuevo modelo de clase apoyado en tecnologías digitales, debe ir acompañado de innovaciones pedagógicas centradas en el modo de trabajo, que involucra los métodos de enseñanza, la organización y selección de actividades. El diseño de las estrategias y las actividades propuestas debe sugerir un esquema de trabajo integrado y conjugado en un marco de enseñanza virtual, que contemple la conectividad a Internet y garantice el avance progresivo e individual del alumno.

Adell (1997) señala que "las aulas virtuales y la educación en línea, a través de las redes informáticas, es una forma emergente de proporcionar conocimientos y habilidades a un amplio sector de la población" (p.10). Por ello, es necesario reestructurar los esquemas referenciales de enseñanza, analizar y diseñar propuestas educativas centradas en secuencias de actividades que contemplen el acceso y la disponibilidad tecnológica de los alumnos.

Las nuevas tecnologías resultan ser un instrumento de mediación en el proceso de enseñanza-aprendizaje que posibilitan un nuevo espacio para la interacción social, donde se puede desarrollar todo tipo de actividades, facilitando la comunicación y la relación interpersonal asincrónica o sincrónica que permiten compartir y debatir ideas, facilitar el trabajo cooperativo y la difusión de las creaciones personales.

Tal como expresan Duart y Sangrá (2000) "los propios estudiantes son los verdaderos protagonistas de su proceso de aprendizaje y los que regulan su propio ritmo de trabajo" (p.16). Esta modalidad virtual de enseñanza crea un modelo dinámico e intencional centrado en el educando, quien desarrolla capacidades que consolidan su formación crítica, autónoma y participativa.

Por tanto, el educando debe tener cierto dominio en el uso de las nuevas tecnologías, conocer las herramientas básicas de su ordenador, presentar destrezas y habilidades específicas para buscar, seleccionar, analizar y comprender la información a la que accede por medio de las nuevas tecnologías.

En este sentido, es fundamental la formación profesional del docente, quien debe incorporar e integrar las nuevas tecnologías en sus planes y procesos formativos, ya que estas tecnologías permiten extender el aprendizaje por fuera de los 
RIIE (2021), Año 12 (15), 44 - 54.

DOI: http://dx.doi.org/10.30972/riie.12155567
Educación: los desafios del aprendizaje en línea y las desigualdades educativas.

límites del aula, son un instrumento de mediación en el proceso de enseñanzaaprendizaje y crean un entorno virtual de enseñanza actualmente predominante que el docente debe conocer.

Los educadores deben generar estrategias de trabajo útiles dentro de estos contextos virtuales que promuevan la construcción de un conocimiento autónomo por parte del educando. Deben lograr establecer un esquema de trabajo que asegure el aprendizaje del alumnado y la construcción significativa de los saberes, considerando las siguientes acciones:

- Guiar y apoyar a los alumnos para que trabajen y piensen por sí mismos, mediante la ejercitación de un pensamiento reflexivo.

- Promover el intercambio entre los estudiantes y favorecer la participación en diversas actividades.

- Posibilitar que los alumnos experimenten el proceso de construcción del conocimiento y la autonomía en el abordaje de los temas y los problemas, implicándose en su análisis y solución.

- Valorar la individualidad y la creatividad del aprendiz.

Para afrontar con éxito esta nueva modalidad virtual y asegurar la continuidad de la enseñanza, las nuevas tecnologías y modos de gestión de clase deben buscar innovar y mejorar la educación.

No se puede pretender únicamente trasladar a la plataforma virtual los materiales y actividades que se utilizaban en el aula presencial, sino que debemos desarrollar apoyos multimedia que integren audio, imagen, texto y, si es posible, que sean interactivas, así como actividades que promuevan el aprendizaje colaborativo mediante herramientas lúdicas, trabajos cuya finalidad sea el desarrollo del pensamiento crítico y el debate, debido a que es necesario atraer al estudiante a este entorno, darle las herramientas y guías necesarias para que desarrolle las actividades y alcancen exitosamente las metas propuestas. (Fernández y González, 2009, p.18).

Estos aprendizajes pueden ser de muy variadas características como asimilación de conocimientos, conceptos y herramientas culturales, como el lenguaje, desarrollo de capacidades de comunicación y expresión, de capacidades para la acción y del sentido práctico, habilidades intelectuales y disposiciones para trabajar con otros y construir nuevas experiencias.

Los educadores tendrán que considerar todos los recursos del sistema, comprendiendo cuáles alternativas sirven mejor para sus propósitos, de acuerdo con su estilo o enfoque personal. Quienes enseñan podrán:

- Analizar y adoptar distintos recursos tecnológicos según las intenciones educativas del proceso de enseñanza.

- Diseñar estrategias educativas que favorezcan la integración de recursos tecnológicos considerando aspectos particulares de los contenidos, los sujetos y los contextos de enseñanza.

- Diseñar de materiales digitales para favorecer el proceso de enseñanza/aprendizaje.

- Construir estrategias propias, considerando las características de los alumnos, el contexto cultural y el ambiente de aprendizaje. 
Como todo proceso de enseñanza, estos recursos permiten la transmisión de conocimientos y el desarrollo de habilidades. Cada uno de ellos puede ser utilizado en forma específica o puede ser combinado con otro, dependiendo de las necesidades educativas. Pero, es importante que los profesores elaboren estrategias concretas apropiadas al contexto socio-cultural e institucional particular, a los recursos tecnológicos, el acceso a Internet, las características y experiencias previas de los alumnos.

\section{La Tecnología y la desigualdad educativa}

El uso de la computadora y la conexión a Internet han contribuido al cambio y desarrollo de diversas áreas como la social, la económica, la ciencia y la educación. Sin embargo, educar en tiempos de pandemia COVID-19 ha evidenciado desigualdad y realidades socioeconómicas preexistentes. La desigualdad se replica y se intensifica en tiempos de pandemia, donde la educación a distancia sólo es posible para aquellos que disponen de los recursos tecnológicos necesarios y se vuelve imposible para otros que carecen de esos recursos. Marchesi (2000) señala que "se puede hablar de igualdad de oportunidades cuando todos los alumnos tienen formal y legalmente las mismas posibilidades educativas" (p.136).

Naciones unidas (2011) afirma que "el uso de las TIC en educación ha beneficiado a una enorme cantidad de personas, facilitando y promoviendo el aprendizaje de diferentes competencias y habilidades acordes con los cambios que las sociedades han sufrido en el último tiempo" (pp. 19-20).

Las Tecnologías de la Información y Comunicación (TIC) en los hogares posibilitan el acceso a la educación en el contexto de pandemia, pero la falta de equidad en el acceso al uso de una computadora, laptop, celular, acceso a internet, software o aplicaciones determinadas y conocimientos previos para utilizar dicha tecnología, refleja una brecha digital en términos de educación. El acceso a internet y el equipamiento tecnológico delimitan el contexto virtual de enseñanza y evidencia la desigualdad de oportunidades entre los estudiantes.

Esta falta de equidad queda totalmente expuesta ante esta situación extrema que se vive en la actualidad por causa de la pandemia COVID-19. Las clases llegan bajo la modalidad virtual sólo a los alumnos que tienen acceso a las nuevas tecnologías con conexión a internet. La continuidad pedagógica durante la pandemia se puede desarrollar de acuerdo con las características de cada institución educativa, de sus docentes y de los recursos tecnológicos de las familias de los estudiantes.

Esta vivencia se conjuga con la elaboración de una agenda educativa en cual se deben exponer propuestas que contemplen estas situaciones de desigualdad. Se debe ofrecer una enseñanza virtual acorde a la tecnología que dispone el grupo-clase, de tal manera que todos los alumnos puedan acceder por igual a su educación.

\section{Objetivos}

En el marco de los planteamientos precedentemente expuestos, se proponen los siguientes objetivos:

- Conocer el equipamiento tecnológico del cual disponen los alumnos considerando sus condiciones socioeconómicas. 
- Evaluar el conocimiento y manejo básico que presenta el grupo clase respecto a las herramientas básicas del ordenador (Word, Excel, PowerPoint)

- Evaluar el conocimiento y las habilidades que presentan los estudiantes para buscar, seleccionar, analizar y comprender la información a la que se accede por medio de las nuevas tecnologías.

\section{Metodología}

\section{Participantes}

Tomando en consideración la modalidad de enseñanza virtual implementada en tiempos de pandemia, se analizaron los conocimientos tecnológicos básicos que presentan 150 alumnos pertenecientes a los cursos de 4to y de 3ro (de ambas divisiones, A y B) de la Escuela Regional de Agricultura, Ganadería e Industrias afines (ERAGIA) de la Universidad Nacional del Nordeste, ubicada en la provincia de Corrientes, Argentina.

\section{Enfoque e Instrumento}

La investigación en la cual se sustenta este trabajo exhibe un alcance descriptivo, en la medida en que se pretende describir y analizar información con respecto al uso de las tecnologías y el aprendizaje en un contexto virtual de enseñanza.

Esta nueva modalidad y gestión de clase establecida a raíz de la pandemia COVID-19 establece un nuevo paradigma de enseñanza, que requiere enfoques adecuados y dentro de secuencias didácticas que fortalezcan la construcción de esquemas cognitivos que posibiliten la adquisición de nuevos conocimientos. Las nuevas tecnologías generarán un cambio en el sistema tradicional de enseñanza, que deberá ser regulada de acuerdo con las condiciones socioeconómicas del grupo-clase.

La propuesta interioriza en este nuevo modelo escolar de enseñanza virtual y las nuevas tecnologías mediante una metodología cuantitativa, a partir de la cual se pretende analizar y recopilar información sobre el uso y manejo que presenta el educando respecto a las nuevas tecnologías. Se indaga acerca del equipamiento tecnológico del que dispone el grupo clase para crear un nuevo esquema de trabajo a futuro que garantice un sistema de enseñanza virtual de calidad, que promueva la construcción de un conocimiento útil, verdadero, significativo y perdurable en el tiempo.

El trabajo de campo consistió en la realización de encuestas a los estudiantes de los cursos de 4to año y de 3er año (en ambos casos, pertenecientes a las divisiones A y B) que asisten a la Escuela Regional de Agricultura, Ganadería e Industrias afines. Se elaboró un cuestionario que comprende los siguientes tópicos:

- Equipamiento tecnológico

- Manejo de herramientas

- Búsqueda en internet

- Competencia y habilidades 
RIIE (2021), Año 12 (15), 44 - 54.

DOI: http://dx.doi.org/10.30972/riie.12155567
Educación: los desafios del aprendizaje en línea y las desigualdades educativas.

\section{Resultados y discusión}

Para lograr una mejor interpretación y poder así anticipar escenarios futuros se han considerado como relevantes las variables que se presentan a continuación, las cuales se corresponden con el contenido de las encuestas realizadas a los estudiantes de 4to año y de 3er año. Se presenta el análisis de las respuestas recogidas a partir de los siguientes resultados, expresados en porcentajes.

\section{Tabla 1.}

Equipamiento tecnológico.

\begin{tabular}{lcc}
\hline & SI & NO \\
\hline Trabajan con su propia & $21 \%$ & $79 \%$ \\
laptop, netbook o PC & $71 \%$ & $29 \%$ \\
Poseen su propio celular y & $71 \%$
\end{tabular}

trabajan con él

Fuente: Elaboración propia

Como se expone en la Tabla 1 , el $21 \%$ de los alumnos encuestados no posee laptop, netbook y PC. Sin embargo, la mayoría dispone de telefonía móvil que serviría como soporte y actuaría como instrumento de mediación y comunicación en el proceso de enseñanza. Por lo cual, los recursos didácticos, las estrategias y las actividades implementadas en las clases virtuales deberán estar adecuados en función a la tecnología que dispone el grupo-clase. Es decir, que la planificación futura de la enseñanza deberá estar ajustada al uso de celulares y ser complementada con uso de netbook, laptop o PC para aquellos alumnos que tienen este equipo tecnológico.

El docente será quien decida: qué enseñar, para qué dominios específicos de conocimiento, cómo enseñar y de qué forma se pueden secuenciar las actividades de acuerdo con el medio tecnológico empleado.

Estas actividades deben:

- Contemplar el acceso y la disponibilidad a internet, considerando las condiciones socioeconómicas del grupo. entre alumnos.

- Facilitar la comunicación asincrónica o sincrónica entre docente-alumnos o

- Permitir compartir y debatir ideas que aseguren la apropiación y la construcción significativa de los saberes.

- Facilitar el trabajo cooperativo y la difusión de las creaciones personales

- Permitir la interpretación, la reflexión y la resolución de problemas de manera individual o grupal.

- Permitir enseñar con contenidos digitales, con entornos de publicación, con materiales multimedia, con videoconferencias, chat.

Todas estas consideraciones permiten crear una modalidad de trabajo competente, basado en un esquema de enseñanza que garantice la efectividad del aprendizaje y el rendimiento escolar de los estudiantes. 
RIIE (2021), Año 12 (15), 44 - 54.

DOI: http://dx.doi.org/10.30972/riie.12155567
Educación: los desafíos del aprendizaje en línea y las desigualdades educativas.

Tabla 2.

Manejo de herramientas

\begin{tabular}{lcc}
\hline & SI & NO \\
\hline Manejo de Word & $23 \%$ & $77 \%$ \\
Manejo de Excel & $5 \%$ & $95 \%$ \\
Manejo de PowerPoint & $12 \%$ & $88 \%$
\end{tabular}

Fuente: Elaboración propia

Se observa que una minoría posee manejo de Word, Excel y Power Point. Por tanto, para lograr optimizar la calidad educativa primeramente es necesario desarrollar una familiarización con el uso de estas herramientas. A este respecto, las actividades deberían permitir:

- El desarrollo de habilidades básicas de escritura y expresión para producir contenido digital en Word.

- la elaboración de gráficas Excel y presentaciones en PowerPoint.

Se trata de actividades altamente productivas que ubican a los estudiantes en un rol más activo y participativo, ya que potencian la creatividad y contribuyen a desarrollar diferentes capacidades que involucran: interpretación y reflexión sobre variables mediante la construcción de gráficas, selección de información y producción creativa mediante la elaboración de PowerPoint, y producción de textos en Word lo cual promueve la lectura, las habilidades de búsqueda y evaluación de la información.

Cada alumno debe realizar un esfuerzo personal para alcanzar la construcción del conocimiento, que requiere de voluntad para incorporar lo nuevo, entender lo que desconocían y construir una estructura que constituye un cuerpo de conocimientos más amplio y profundo.

Tabla 3.

Búsqueda en Internet

\begin{tabular}{lll}
\hline & SI & NO \\
\hline Se conectan a Internet & $26 \%$ & $74 \%$ \\
para buscar Información & &
\end{tabular}

Fuente: Elaboración propia

Un bajo porcentaje de alumnos se conecta a Internet para buscar información. Este dato nos permite visualizar el escenario escolar en cuanto a la búsqueda de información y la falta de conectividad en un marco desigual de acceso, que limita el contexto virtual de enseñanza y pone en evidencia la realidad socioeconómica diversa del grupo-clase, donde al parecer solo unos pocos tienen acceso a Internet.

Esta desigualdad retrasa el aprovechamiento de TIC y la disponibilidad de software y aplicaciones, el acceso a la alfabetización digital vinculado con la educación para adquirir habilidades digitales, que implica búsqueda, selección, organización, comprensión y valoración de la información. Todas estas habilidades son necesarias, ya que permitirán establecer competencias en entornos de aprendizajes abiertos, en contextos de incremento y dinamismo de la información. 
RIIE (2021), Año 12 (15), 44 - 54.

DOI: http://dx.doi.org/10.30972/riie.12155567
Educación: los desafios del aprendizaje en línea y las desigualdades educativas.

La búsqueda y consulta de información permitirían obtener un alto grado de interdisciplinariedad debido a la gran variedad de información disponible a través de los enlaces hipertextuales y buscadores, para ello el aprendiz deberá crear criterios y estrategias de búsqueda y selección de la información. Es decir, que los alumnos apoyados en la tecnología podrán buscar información y desarrollar su juicio crítico y su iniciativa de aprender continuamente todo aquello que sea esencial durante el proceso de aprendizaje para cumplir con las intenciones formativas y desarrollar su autoaprendizaje. De ahí que los estudiantes asuman el rol de constructores y gestores de su propio aprendizaje.

El uso de Internet permitiría el acceso a chat y a los foros, propiciando actividades colaborativas, estimulando el intercambio, el debate y los trabajos conjuntos entre los alumnos como fuente de conocimiento, más allá del profesor. Además, favoreciendo el aprendizaje social, la construcción de sus experiencias, valorando sus esfuerzos y fortaleciendo su autonomía.

En cualquiera de estos niveles, el aprendizaje representa una modificación o un cambio en el comportamiento del sujeto, porque éste relaciona la información y los significados del contenido sobre el que trabaja, la vincula con sus conocimientos, significados y experiencias previas, y por ello, la comprende. Esto demanda una intensa actividad participativa por parte de quienes aprenden.

\section{Tabla 4.}

Competencia y habilidades

\begin{tabular}{lcc}
\hline & SI & NO \\
\hline Saben colocar el nombre a un archivo. & $20 \%$ & $80 \%$ \\
Ordenar archivos en carpetas. & $15 \%$ & $85 \%$ \\
Tienen dirección electrónica y lo usa & $11 \%$ & $89 \%$ \\
habitualmente. & $10 \%$ & $90 \%$ \\
$\begin{array}{l}\text { Usan distintas aplicaciones del celular para } \\
\text { leer, enviar archivos (documentos, planillas de }\end{array}$ & & \\
$\begin{array}{l}\text { cálculo, etc.). } \\
\text { Usan las aplicaciones gratuitas de Google. }\end{array}$ & $0 \%$ & $100 \%$ \\
$\begin{array}{l}\text { Usan WhatsApp preferentemente para enviar } \\
\text { mensajes }\end{array}$ & $100 \%$ & $0 \%$
\end{tabular}

Fuente: Elaboración propia

Conforme a lo expuesto en la tabla, solo un pequeño porcentaje de estudiantes cuenta con competencias y habilidades para nombrar archivos y ordenar por carpeta. La mayoría no dispone de un correo electrónico, que constituye un sistema básico de comunicación en Internet que permite recibir y enviar mensajes con archivos adjuntos (lo cual facilitaría la comunicación y las relaciones interpersonales asincrónicas). Tampoco utilizan las distintas aplicaciones del celular para leer o enviar archivos, ni las aplicaciones de Google.

La interpretación de estos resultados nos permite recabar información necesaria para controlar del ritmo de la enseñanza y las secuencias que marcan el aprendizaje de acuerdo con las necesidades educativas individuales. Ante esta situación, es necesario idear estrategias metodológicas que requieren de una 
alfabetización tecnológica continua para que los alumnos puedan acceder y conducirse de manera competente a través de la cultura digital.

Por otro lado, se observa que todos los alumnos utilizan WhatsApp. Esta aplicación serviría como medio para la comunicación de forma simultánea y en tiempo real, con el docente y entre compañeros. Permitirá el intercambio de opiniones y conocimientos, fomentando el desarrollo de habilidades expresivas y procesos de comunicación ágiles que se orientan al logro de acuerdos y resoluciones de forma rápida y puntual.

Las afirmaciones, las sugerencias y las opiniones, deben estar justificadas mediante argumentaciones y razones, a fin de que puedan ser criticadas de forma constructiva por el resto de los miembros del grupo. Es importante ir acordando y fijando un sistema de intercambio de información constante que permita la elaboración, organización y revisión progresiva de toda la información. Este intercambio es acumulativo, el grupo discute para construir un conocimiento común.

Además, se podría enviar archivos (de lectura o actividades) y compartir videos con contenidos temáticos estructurados en función de un objetivo o eje central, que va a depender de la naturaleza del contenido, del pensamiento del docente y del proceso de aprendizaje que se considere relevante para el alumno.

Cada medio didáctico ofrece determinadas posibilidades de utilización en el desarrollo de las actividades, sirve como soporte y actúa como instrumento de mediación para acceder al material de trabajo que debe estar integrado a la "plataforma-entorno-aprendizaje" para aprovechar las funcionalidades del medio tecnológico empleado. Hay que tener en cuenta que estos medios no solo transmiten información, sino que también son mediadores entre la realidad y los estudiantes. No siempre se dispone de la infraestructura que requieren ciertos medios, ni los estudiantes presentan las habilidades necesarias para utilizar las nuevas tecnologías.

El educador puede desarrollar múltiples formas de trabajo, en virtud de sus objetivos y atendiendo a la realidad de sus alumnos. Puede enseñar con contenidos digitales, con entornos de publicación, con redes sociales, con materiales multimedia, con videoconferencias, chat o foros, con trabajos colaborativos, etcétera.

En cualquier caso, deben existir criterios de selección y organización de la información para favorecer la construcción de saberes y el pensamiento reflexivo, conjugados en un marco de enseñanza que garantice el avance progresivo y el desarrollo individual del alumno en contextos virtuales de enseñanza.

\section{Conclusión}

La aparición de la pandemia producida por el Covid-19 produjo cambios profundos en distintos sectores de la sociedad. En el plano educativo, la interrupción de las formas de cotidianeidad escolar generó la readaptación y el ajuste de la enseñanza en entornos virtuales en tiempo de pandemia, que requirieren de una planificación basada en el desarrollo individual y en la expansión de habilidades que propicien un escenario óptimo en el proceso de enseñanza-aprendizaje.

Tanto docentes como alumnos requieren de una alfabetización tecnológica para que puedan acceder y conducirse de manera competente a través de la cultura y la tecnología digital de acuerdo con sus necesidades educativas individuales, lo que 
implica desarrollar autonomía e iniciativa para emprender y autodirigir procesos de aprendizaje personales, comunicar ideas con claridad y precisión por medio de una variedad de recursos tecnológicos.

El acceso a Internet, el equipamiento tecnológico y el contexto socioeconómico delimitan el marco virtual de enseñanza. En tal sentido, las herramientas digitales que se promueven en la educación a distancia se basan en una lógica individual y técnica. Por lo que se requiere de estrategias de trabajo útiles que promuevan la construcción de un conocimiento autónomo y efectivo que favorezca la integración de una serie de conocimientos, habilidades y actitudes adecuadas a la realidad de cada estudiante.

La planificación y la selección de las distintas estrategias involucradas en el proceso de aprendizaje en entornos virtuales, requieren de un esquema de trabajo que garantice un sistema de enseñanza que permita integrar instrumentos, estructuras y funciones que promueva la eficacia de la acción docente y que contemple las condiciones socioeconómicas y tecnológicas de los sectores más vulnerables, para que todos los alumnos puedan acceder a una educación a distancia de calidad y construir un conocimiento útil, verdadero, significativo y que perdure en el tiempo.

\section{Referencias bibliográficas}

Delgado Fernández, M., y Solano González, A. (2009). Estrategias didácticas creativas en entornos virtuales para el aprendizaje. Revista Electrónica Actualidades Investigativas en Educación, 9 (2),1-21. Recuperado de https://www.redalyc.org/pdf/447/44713058027.pdf

Adell, J. (1997). Tendencias en educación en la sociedad de las tecnologías de la información. Edutec-e. Revista Electrónica de Tecnología Educativa, (7), 1-21. https://doi.org/10.21556/edutec. 1997.7.570

Abreu, J. (2020). Tiempos de Coronavirus: La Educación en Línea como Respuesta a la Crisis. Daena: International Journal of Good Conscience,15 (1), 1-15. Recuperado de http://www.spentamexico.org/v15-n1/A1.15(1)1-15.pdf

Duart, J., y Sangrá, A. (2000). Formación universitaria por medio de la web: un modelo integrador para el aprendizaje superior. En J. Duart y A. Sangrá (Comps.) Aprender en la virtualidad (pp. 23-50). Barcelona, España: Gedisa.

Marchesi, A. (2000). Un sistema de indicadores de desigualdad educativa. Revista Iberoamericana de Educación, mayo-agosto (23),135-163. Recuperado de https://www.redalyc.org/pdf/800/80002305.pdf

Naciones Unidas (2011). Desafíos para una Educación con Equidad en América Latina y El Caribe. Encuentro Preparatorio Regional 2011 Naciones Unidas. Consejo Económico y Social. Revisión Ministerial Anual. ECOSOC RMA. CEPAL. Buenos Aires, Argentina, 8, 1-34.

Sunkel, G., Trucco, D., y Möller, S. (2011). Aprender y enseñar con las tecnologías de la información y las comunicaciones en América Latina: potenciales beneficios. Series políticas sociales, 169, 1-67. Santiago de Chile: Naciones Unidas. 